\title{
What is the Link Between Emotional Intelligence and Emotion Regulation? Behavioural and Resting-State Functional Connectivity Evidences
}

\section{Federico Zanella}

University of Trento

Bianca Monachesi ( $\nabla$ bianca.monachesi@unitn.it )

University of Trento

Alessandro Grecucci

University of Trento

\section{Research Article}

Keywords: Emotion Regulation, Emotional Intelligence, Independent Component Analysis, unsupervised machine learning, resting state networks

Posted Date: January 25th, 2022

DOI: https://doi.org/10.21203/rs.3.rs-1276773/v1

License: (c) (i) This work is licensed under a Creative Commons Attribution 4.0 International License. Read Full License 


\section{Abstract}

A converging body of behavioural findings supports the hypothesis that the dispositional use of Emotion Regulation (ER) strategies depends on trait Emotional Intelligence (trait EI) levels. Unfortunately, neuroscientific investigations of such relationship are missing. To fill this gap, we analysed behavioural and resting state data from 79 healthy participants to corroborate whether the same neural circuit predicting trait El, also predicts specific ER strategies. An unsupervised machine learning approach (Independent Component Analysis) was used to decompose resting-sate functional networks and to assess whether they predict trait El and specific ER strategies. Behavioural results showed that total trait El index significantly predicts and negatively correlates with the frequency of use of typical dysfunctional ER strategies (suppression and self-blame). Crucially, we observed that an increased BOLD temporal variability within sensorimotor and language networks predicts both high trait El and the frequency of use of cognitive reappraisal. By contrast, a decreased variability in language network predicts the use of suppression. These findings support the tight connection between high trait $\mathrm{El}$ and individual tendency to use functional ER strategies, and provide the first evidence that modulations of BOLD temporal variability in specific brain networks may be pivotal in explaining this relationship.

\section{Introduction}

Emotion Regulation (ER) is employed to modify current emotional states and adaptively respond to the environment ${ }^{1-4}$. Not surprisingly, difficulties in ER are involved in compromised well-being and mental health ${ }^{5,6}$. Namely, the selection of specific strategies and the excessive or rigid usage of them has been associated with negative outcomes ${ }^{7-9}$ contributing to a general distinction between dysfunctional and functional strategies. Among the former, some studies reported that suppression (i.e., the inhibition of the expressive reactions to the emotional events), fails to engender subjective relief after experiencing negative emotions, it comes with several costs in terms of physiological, cognitive and social functioning $5,10,11$, and it has been associated with decreased well-being ${ }^{12}$ and several psychopathologies (e.g., depression and anxiety disorders ${ }^{13,14}$. Differently, reappraisal (i.e., the ability of cognitively changing the impact of the emotional event) has been generally considered an adaptive strategy, associated with healthiness and personal satisfaction ${ }^{5}$ and that successfully modulates the affective state ${ }^{15}$ emotionrelated peripheral physiological indexes ${ }^{16}$ as well as neural activity ${ }^{17}$.

The simplistic distinction between functional and dysfunctional strategies, however, risks to conceal the role of individual differences in selecting and recurrently using regulation strategies ${ }^{18}$. What leads someone to use one given strategy or to use it more often than others? Even though individuals' dispositions may be crucial to comprehend the variability behind the selection and usage of emotion regulation strategies ${ }^{19}$, investigations in this sense are still scant ${ }^{18,20}$. Only recently, an interesting line of research suggests a link between ER style and the construct of Emotional Intelligence ${ }^{18,21}$. El refers to how people experience, understand, and manage emotions both in terms of individuals' abilities (ability El) and, crucially for the present study, in terms of personal traits (trait El), that is, on the basis of 
individuals' perception of emotional events ${ }^{22}$. Some studies reported that the ability El fails in predicting specific emotion regulation processes ${ }^{23}$, and when it does, it has to be coupled with perceived competence (trait El) to comprehend the beneficial consequences of the adaptive response ${ }^{24}$. Moreover, similar levels of ability El may results in adaptive as well as deviant responses ${ }^{25,26}$. The measure of traits $\mathrm{El}$, then, seems more promising to explain how specific ER processes are selected. More directly, behavioural studies reported that individuals with high levels of trait El tend to use contextually appropriate regulation strategies, whereas individuals with low levels of El adopted non-adaptive adjustment styles ${ }^{18,27}$. However, the causal relationship between trait El and ER, the nature and the extent of their touchpoint remain unclear, especially in neural terms. The present study, then, aims to corroborate the influences trait EI has on ER style, and to test the hypothesis that their link relies on a shared neural substrate. Indeed, a few neuroimaging studies dealing with trait El ${ }^{28-30}$ seem to suggest at least a partial neural overlap with findings from neuroimaging studies of ER ${ }^{31-34}$. ER processes rely on the activity in brain areas related to both emotion processing (limbic system) and high executive functions (prefrontal regions) ${ }^{31-36}$. Moreover, structural studies found a correlation between specific ER style and grey matter alterations in networks including fronto-parietal ${ }^{37}$ and subcortical ${ }^{38}$ regions. Similarly, Killgore and Yurgelun-Todd ${ }^{28}$ found that during presentation of emotional faces, subjects with higher levels of trait EI showed lower activity in limbic and paralimbic regions. More on this, Kreifelts and colleagues ${ }^{29}$ reported a correlation between traits El and activity in cortical regions underpinning emotion processing, although they did not find correlation with activity in more subcortical, emotion-related regions (e.g., amygdala, see also ${ }^{39}$ for similar null results). From a structural neuroimaging perspective, it has been shown that grey matter alterations in fronto-limbic brain areas correlates with traits $\mathrm{El}^{30}$.

Besides structural and task-related functional evidence, and more importantly for the present study, some authors relied on resting-state functional connectivity (RS-FC) to understand the neural signature of ER and EI. RS-FC is a valuable source to understand the neural mechanisms behind several psychological states ${ }^{40-42}$, as well as emotional processes ${ }^{17,31}$. In addition, altered functional connectivity appears to be associated with many psychopathologies ${ }^{43,44}$. Evidence from resting-state studies showed that higher trait El scores was associated with higher activity in the inferior frontal gyrus (IFG) and in the inferior parietal lobule (IPL), and with less activity in regions of the insula, cingulum, ventromedial prefrontal cortex (vmPFC), amygdala, hippocampus, parahippocampal gyrus ${ }^{46}$, and fusiform gyrus ${ }^{45}$. Moreover, Takeuchi and colleagues ${ }^{47}$, showed that trait El score positively correlated with resting-state activity between medial prefrontal cortex (mPFC) and the precuneus, the intracalcarine cortex, and between anterior insula (aIC) and the right portion of the dorsolateral prefrontal cortex (dIPFC). In ER context, Pérez and colleagues ${ }^{48}$ reported a negative correlation between the connectivity of the right basolateral amygdala, the left insula, and the supplementary motor cortex (SMA) with the frequency of use of cognitive reappraisal, and a positive correlation between emotion suppression strategy and the activity of the right basolateral amygdala, the dorsal anterior cingulate (dACC), the supplementary motor cortex (SMA), and in the left medial portion of the amygdala. In contrast, Uchida and colleagues ${ }^{49}$ reported a negative correlation between functional connectivity in the amygdala and medial prefrontal cortex 
(mPFC) with the success of applying the cognitive reappraisal strategy. It is important to note also that Dörfel and colleagues ${ }^{50}$, failed to replicate and extend both the previously mentioned studies.

Beside inconsistencies, the extant literature makes it reasonable to hypothesize a neural overlap between El and ER networks. Notably, the inconsistencies in ER and El studies, may derive from some methodological issues. Indeed, the majority of studies used massive univariate approaches, and a priori selected regions $28,28,34,45,48,50$. Widely distributed processes, such as ER or El, may be better captured using multivariate approaches and a network perspective ${ }^{51}$. For this reason, in the present study, we adopted a whole brain data-driven approach (Independent Component Analysis, ICA) that allows us to identify the variations of BOLD signal activity over time within major brain networks rather than considering signals from a priori decided regions of interest. ICA, being a blind source separation method, is an unsupervised machine learning approach able to identify non-overlapping independent neural circuits $^{52}$. Such independent circuits represent meaningful naturally separated circuits that bypass anatomically and histologically based regions, and have the advantage of reducing brain complexity into low dimensional spaces ${ }^{51}$. The BOLD temporal variability (SD BOLD) for every resting-state network was used to predict tEI and ER strategies. BOLD variability is defined as a fluctuation in neural activity over time, and an increment in this measure reflects greater functional network complexity and is associated with more effective information integration ${ }^{53-55}$. According to Moreira and colleagues ${ }^{56}$, this feature is particularly relevant for psychological phenomena that develop over time, such as $\mathrm{ER}^{57,58}$, or affective states ${ }^{59}$ and represents an index of the degree of cognitive flexibility ${ }^{60}$.

Building on the above considerations, the present study is aimed to support and extend previous behavioural findings on the role of trait El in determining ER strategies, and to disclose which overlapping neural network may subserve this relationship. We hypothesize that the total level of trait El as well as the score of its subscales (measured by the TeiQueSF questionnaire, ${ }^{61}$ significantly predict the use of different emotional regulation strategies (measured by the ERQ, ${ }^{12}$, and CERQ questionnaires, ${ }^{62}$ ) in the way that the higher the trait El and/or subscales scores, the higher the usage of ER strategies considered as functional (reappraisal, acceptance, etc), or, on the other side, the lesser the usage of some ER strategies considered as dysfunctional (self-blame, catastrophizing, etc). Then, we delineate the networks underlying El using an ICA approach on the resting-state functional connectivity, and we assess whether these networks may predict the dispositional use of different ER strategies. We expect to find that the activity in regions responsible for the processing and control of socio-emotional information (e.g., insula, frontal and parietal regions) predicts both the individual differences in El (as measured by TeiQueSF), as well as the ER usage (measured by ERQ and CERQ). In conformity of the method and neural measure used, these areas will be discussed in terms of common resting state networks.

\section{Results}

\section{Behavioural Results}


The total El trait index significantly predict and is negatively associated (negative $\beta$ values) with the frequency of use of the following strategies: Suppression (ERQ) $\left(F(1,78)=9.705, R^{2}=0.11, p=0.003 ; \beta=\right.$ -0.335 $p F D R=0.022)$; Self-blame (CERQ) $\left(F(1,78)=7.867, R^{2}=0.093, p=0.006 ; \beta=-0.304, p F D R=0.022\right)$; and Catastrophizing (CERQ) $\left(F(1,78)=8.742, R^{2}=0.10, p=0.004 ; \beta=-0.319, p F D R=0.022\right)$. For what concerns the four TEIQue-SF factors, Emotionality $(\beta=-0.339, p F D R=0.004)$, and Well-being $(\beta=-0.239, p F D R=0.01)$ predict Suppression (ERQ) $\left(F(4,75)=4.908 \mathrm{R}^{2}=0.21, p=0.001\right)$; Self-control $(\beta=-0.377, p F D R=0.004)$, Emotionality $(\beta=0.343, p \mathrm{FDR}=0.004)$, and Well-being $(\beta=-0.339, p \mathrm{FDR}=0.03)$ predict Rumination (CERQ) $\left(F(4,75)=5.332, R^{2}=0.27, p<0.005\right)$; Self-control $(\beta=-0.269, p F D R=0.03)$, and Well-being $(\beta=-0.332$, $p \mathrm{FDR}=0.01)$ predict Catastrophizing (CERQ) $\left(F(4,75)=4.258, R^{2}=0.21, p<0.001\right)$; Self-control $(\beta=-0.33$, $p F D R=0.02)$ predicts Blaming others (CERQ) $\left(F(4,75)=2.24, R^{2}=0.12, p=0.042\right)$. To conclude, as can be noted from the negative $\beta$ values of the predictors, the lower is the El, the higher is the individual tendency to use non-adaptive ER regulation strategies such as Rumination, Blaming others, Catastrophizing, Suppression.

\section{Functional Connectivity Results}

Multiple Regression analyses (stepwise) showed that the BOLD variability of IC20 $(\beta=0.295$, $p \mathrm{FDR}=0.008)$ significantly predicts total El index $\left(F(1,77)=7.335, r=0.36, R^{2}=0.08, p=0.008\right)$; the BOLD variability of IC2 $(\beta=-0.252, p F D R=0.025)$ significantly predict Self-control $(F(1,77)=5.225, r=0.25$, $\left.R^{2}=0.106, p=0.025\right) ; \mathrm{IC} 18(\beta=0.360, p \mathrm{FDR}=0.003), \mathrm{IC} 16(\beta=0.239, p \mathrm{FDR}=0.03)$, and IC1 $(\beta=0.208$, $p F D R=0.045)$ predict Sociability $\left(F(3,75)=10.074, r=0.458, R^{2}=0.210, p=0.001\right)$; finally, IC20 $(\beta=0.380$, $p F D R=0.003)$, IC1 $(\beta=-0.309, p F D R=0.01)$, and IC9 $(\beta=0.235, p F D R=0.03)$ predict Well-being $\left(F(3,75)=5.734, r=0.432, R^{2}=0.187, p=0.001\right)$. To summarize, with the exception of IC1 and IC2 (whose $\beta$ are negative predictors of respectively wellbeing and self-control factors) the more is the BOLD variability in the others ICs, the higher is the trait El level. The brain networks identified by the IC's includes clusters of cortical and subcortical regions at a cluster significance level $p<0.05$ ( $p$ FDR corrected) and voxel significance $p<0.001$ ( $p$ FDR corrected). Based on CONN's correlational spatial match to template approach, the identified ICs are attributable to known resting state networks: IC20 and IC16 = sensorimotor network; IC1 = cerebellar network; IC2 and IC9 = language network; IC18 = visual network.

Among the IC's that were significantly predictive of trait El, the BOLD variability of IC20 $(\beta=0.494$, $p F D R=0.005)$ predicts Cognitive Reappraisal $(\mathrm{ERQ})\left(F(6,72)=3.224, R^{2}=0.21, p=0.007\right)$. Also, the BOLD variability of IC20 $(\beta=0.351, p \mathrm{FDR}=0.03)$, IC16 $(\beta=-0.282, p \mathrm{FDR}=0.01)$ and IC9 $(\beta=0.299, p \mathrm{FDR}=0.01)$, predict Positive Reappraisal (CERQ) $\left(F(7,71)=3.145, R^{2}=0.23, p=0.006\right)$. Finally, the BOLD variability of IC9 $(\beta=-0.360, p F D R=0.01)$ predicts Suppression (ERQ) $\left(F(6,72)=2.302, R^{2}=0.16, p=0.043\right)$.

As can be noted from the positive $\beta$ values, the higher is the BOLD variability the higher is the frequency of use of Cognitive Reappraisal and Positive Reappraisal (with the exception of IC16). Whereas, the lower is the BOLD variability the higher is the frequency of use of the Suppression. The brain networks 
identified by the IC20, IC16 and IC9 include clusters of cortical and subcortical regions (see Table 1 for details) at a cluster significance level $p<0.05$ ( $p$ FDR corrected) and voxel significance $p<0.001$ (pFDR corrected) (See Fig. 1).

Table 1

Regions identified in the independent component IC20, IC16, IC9. Clustering indicates the spatial distribution of ROls at the cortical and subcortical levels. Cluster threshold at $\mathrm{p}<0.05$ ( $p F D R$ corrected) and voxel threshold $p<0.001$ (pFDR corrected, two sided). For each cluster we selected the brain regions with the highest covering proportion (\%) of Harvard-Oxford Atlas ROI. Peak are reported in MNI coordinates. $\mathrm{L}=$ Left; $\mathrm{R}=$ Right; $\mathrm{BA}=$ Brodmann Area.

\begin{tabular}{|c|c|c|c|}
\hline IC & Peak & ROI (Harvard, Oxford Atlas) & p-valueFDR \\
\hline \multirow[t]{7}{*}{ IC20 } & $-18+30+60$ & Supplementary Motor Cortex & $p<0.005$ \\
\hline & $-58-18+32$ & R Supramarginal Gyrus & $p<0.005$ \\
\hline & $-60-20+30$ & L Supramarginal Gyrus & $p<0.005$ \\
\hline & $+34-22+20$ & R Hippocampus & $p<0.005$ \\
\hline & $+48+04+26$ & R Inferior Frontal Gyrus & $p<0.005$ \\
\hline & $-42-02-08$ & Insular Cortex & $p<0.005$ \\
\hline & $+34-22+20$ & L Cerebellum (BA 4-5) & $p<0.005$ \\
\hline \multirow[t]{6}{*}{ IC9 } & $+18+08+14$ & Thalamus L & $p<0.005$ \\
\hline & $+66-30+12$ & R Superior Temporal Gyrus & $p<0.005$ \\
\hline & $-64-34+14$ & L Superior Temporal Gyrus & $p<0.005$ \\
\hline & $+10-70-40$ & Vermis (BA 8) & $p<0.005$ \\
\hline & $+14-90+00$ & L Intracalcarine Cortex & $p<0.005$ \\
\hline & $-20-36-42$ & L Cerebellum (BA 10) & $p<0.005$ \\
\hline \multirow[t]{5}{*}{ IC16 } & $-56-04+24$ & Supplementary Motor Cortex & $p<0.005$ \\
\hline & $-64-36+44$ & Supramarginal Gyrus & $p<0.005$ \\
\hline & $+18+18-12$ & L Middle Temporal Gyrus & $p<0.005$ \\
\hline & $+20-66-38$ & Cerebellum (BA 8) & $p<0.005$ \\
\hline & $-22-68-40$ & Cerebellum (BA 7) & $p<0.005$ \\
\hline
\end{tabular}

\section{Discussion}

ER processes are daily employed by individuals to modify the emotional states they are experiencing. However, the individuals' variability to use one or other strategy is still poorly understood. How can such 
variability be explained? In the present study we investigated which aspects of trait El may predict specific ER strategies usage. We were also interested in finding the neural bases associated with $\mathrm{El}$, by analysing the functional connectivity of naturally grouping circuits decomposed by an unsupervised machine learning approach (ICA). One intriguing question, was whether El and ER share at least some neural bases. This would be an additional proof of their intimate relationship.

At a behavioural level, we confirm and extend previous studies suggesting a relationship between low trait El and maladaptive emotion regulation processes ${ }^{18,27}$. Indeed, we found that individuals with low levels of trait El are predisposed to use typical non-adaptive ER style (i.e., suppression, self-blame, and catastrophizing). In particular, low scores in the subscale of "self-control" predispose to blaming others and rumination. Low scores in the "well-being" subscale predispose to the use of suppression, rumination, and catastrophizing. Finally, low scores in "emotionality" predispose to the use of suppression and rumination. In a complementary way, functional connectivity results showed that increased BOLD variability in the sensorimotor network identified by the IC20 predicts high score in the total traits El index. Modulations of BOLD variability in the same and other networks spanning from the visual, language and cerebellar ones also predicted the four subscales of trait El. Namely, modulations of BOLD variability in visual, sensorimotor (related to IC16), and cerebellar networks predicted high scores in the subscale of sociability. High score in wellbeing subscale was predicted by increased and decreased bold variability in sensorimotor (related to IC20) and cerebellar networks, respectively. Finally, decreased BOLD variability in language network predicted self-control subscale. Most importantly for the present study, increased BOLD variability in network related to sensorimotor (when identified by the IC20 but not the IC16) and language also predicted the use of reappraisal strategy, as measured by both ERQ and CERQ questionnaires. Interestingly, decreased BOLD variability in the language network also predicted the use of emotion suppression strategy.

Rumination and suppression, the two strategies more often predicted by the subscales of trait El in the present study, have been usually related to negative outcomes and psychopathologies ${ }^{5}$. Likewise, low level of traits El are also associated with psychopathological disorders ${ }^{63,64}$. The link between rumination and "self-control", which is associated with difficulties in managing stressful situations and impulsive behaviours, is supported by recent literature suggesting that impulsivity plays a critical role in rumination, and stressor mediated this association ${ }^{65}$. Low self-esteem (a relevant aspect in the subscale of "wellbeing" along with the feelings of dissatisfaction) emerged as an important predictor of rumination ${ }^{66}$, and it has been indirectly linked to suppression since involved in shame, which has been already coupled with this regulation strategy ${ }^{67,68}$. In addition, low emotionality and the related difficulty in emotions recognition and expression ${ }^{61}$, may lead individuals to use suppression that occurs late in the process of emotion generation ${ }^{69}$. The trait El subscale of "sociability" surprisingly did not predict any emotion regulation strategy. This null result can be explained by behavioural evidence showing that low sociability is not associated with high psychological reactivity, negative emotional intensity, dispositional negative affect, and personal distress, as shyness for example does. Low sociability is rather associated with low social support seeking ${ }^{70}$, strategy which may have escaped the taxonomy used in the ERQ and CERQ. In 
support of that, trait El predict social sharing when this latter is included in the set of regulation strategies $^{23}$.

At a neural level, we enriched our understanding of the neural overlap between trait El and ER, by showing that two different networks (sensorimotor and language ones) involved in the former, are also involved in the latter. Among areas of the IC20-related sensorimotor network, the inferior frontal gyrus emerged in previous resting-state study on trait $\mathrm{El}^{45}$ and it was interpreted as a part of a circuit related to social and emotional processing. A positive correlation between the activity of frontal regions and trait El has also been found by Takeuchi and colleagues ${ }^{71}$. On the other hand, the supplementary motor cortex (SMA) and the cerebellum positively correlated with the trait $\mathrm{El}^{45}$, playing a critical role in cognitive control mechanisms. The somatosensory cortex, and its portion of the supramarginal gyrus (SMG) are involved in the recognition of emotions, the understanding of the emotional states of others ${ }^{72}$, and are more generally part of the mirror neuron system ${ }^{73,74}$. Damasio and colleagues ${ }^{75}$ observed a change in activity in the somatosensory cortex during a task in which subjects reflected on experiences that generated different emotional states. An activation of the somatosensory cortex was also observed in tasks of interoceptive awareness, suggesting that this cortical structure may be involved in the awareness of internal states ${ }^{76}$. Finally, insula is deemed to facilitate social interaction, and decision making by integrating sensory, affective, and bodily information, and it has been traditionally reported as neural correlate of trait $\mathrm{El} 47,77,78$. The functional implications of these areas in socio-emotional processing and cognitive control ${ }^{45,79}$ make intuitive their involvement also in control-related ER processes. Another intriguing finding, indeed, is that the same circuit predicting trait El, also predicts specific adaptive ER strategy. For example, there is evidence of a correlation between the functional connectivity of the left insula, supplementary motor cortex (SMA) ${ }^{48}$, and inferior frontal gyrus ${ }^{80}$ with the frequency of use of reappraisal. Generally, these areas are implicated in the well-established network underlying cognitive control of emotions by reappraisal ${ }^{32,36}$. In light of this finding, the negative correlation between the BOLD variability in the IC16-related sensorimotor network and the positive reappraisal may seem incoherent at first sight. However, this result is in line with and corroborate the behavioural ones which shows no association between the trait EI Sociability scale and ER strategies. Indeed, it is worth to bring back that, differently from the IC20, which significantly predicted the total trait El and the well-being scale, the IC16 predicts the Sociability scale and this may explain why this component yield a negative correlation with ER in neural terms. In addition, how figure 1 shows, the two sensorimotor-related components involved overlapping, but still different brain regions.

Among the other El-related networks, our study showed that also the language network is shared with ER style. More specifically, the BOLD variability in this network positively correlates and predicts adaptive (reappraisal) strategy, and negatively correlates with maladaptive (suppression) strategy. Although this result is less common in neuroscientific literature, recent studies highlight that mechanisms related to language and semantic processing may be shared by several emotion regulation strategies ${ }^{81}$. Other works provide more direct evidence that language can increase the discreteness of an emotion 
experience, facilitating its regulation ${ }^{82,83}$. Brain regions involved in semantic processing (i.e., temporal pole) are also functionally connected with sensory processing regions (i.e., sensorimotor area) providing representational content for emotions ${ }^{84}$. Further, activation of language areas (e.g., bilateral temporal gyrus) has been associated with adaptive emotion regulation strategies in which verbal labelling of affective state represents a productive way to reach self-awareness, but, critically, these areas were not involved in suppression strategy ${ }^{85}$. This is in line with the negative correlation we found.

The relation between BOLD variability, trait El and adaptive vs maladaptive emotion regulation strategies can be better understood coming back to what the BOLD variability means. Several studies point out that greater BOLD variability positively influences adaptability, flexibility and efficiency of neural system in response to the multiplicity and uncertainty of environmental stimuli ${ }^{53-55,86}$. As a such, it is reasonable that cognitive and affective mechanisms related to the functionally connected regions are better implemented by individuals showing increased temporal variability in the network ${ }^{87}$. Accordingly, our findings suggest that the increased BOLD variability in the sensorimotor and language networks play a critical role in predicting both high level of trait El and adaptive emotion regulation strategy, in terms of a better social and emotional information integration, self-awareness along with a more efficient cognitive control. That temporal variability in these networks significantly predicts high traits El and the frequency of use of Cognitive Reappraisal strategy is explained as an adaptive feature of the neural response, allowing the brain to easily access different " states", required to complete cognitive tasks ${ }^{88,89}$. By the same token, we could also infer that less variability in language network could imply a difficulty of the subjects to process emotional information resulting in the maladaptive emotion suppression strategy. Then, greater temporal variability may represent a neural predisposition marker which facilitate individuals in the stages underlying the dynamic process of emotional regulation, identification, selection, and implementation ${ }^{90}$. This finding provides a context for and corroborate the hypothesis that regulation strategies and their outcome may depend on factor such as the individual differences. Neural flexibility and adaptability increase perception and control of the emotional event determining at the same time the success of an emotion regulation process ${ }^{7}$. Importantly, resting-state functional connectivity may be pretty helpful to investigate task-independent constructs ${ }^{91}$ such as those related to personal traits are.

Besides these new findings, the study has some limitations to point out. While the data-driven approach allowed us to consider the activity of the whole brain and the role of naturally grouping circuits, theory driven analyses (i.e., Dynamic Causal Model) that may facilitate inferences with respect to specific brain regions, or to identify causal relationships between them, may be a valuable and complimentary alternative. In addition, the discissed networks as identified by the spatial match included portions of salience and executive networks. Future studies are needed to explore the contributions of such networks to both El and ER. Finally, building on the existing literature, we focused on trait El. However, other aspects of El may be worth to be investigated.

To conclude, the present findings reveal that the role of trait El in predicting adaptive ER style relies on a shared and more efficient functional connectivity network involved in social and emotional information 
processing to understand self and others' affective states, and in top-down mechanisms which contribute to the control of emotions. Consequently, our study not only provide further support to the causal relationship between traits El and maladaptive ER strategies, but is also represents a first step to understand the neural mechanisms able to explain this relationship. Increased variability of the BOLD signal within a sensorimotor and language networks is a mainstay for the neural structure of high traits $\mathrm{El}$ and at the same time predisposes to the use of adaptive emotional regulation strategy. By contrast, a decreased variability in language network predisposed to the use of a maladaptive emotion regulation strategy.

\section{Methods}

\section{Participants}

The data analysed in this study were selected from the open-source dataset "Max Planck Institute Leipzig Mind-Brain-Body Dataset LEMON" 92 . Subjects were recruited by researchers at the University of Leipzig, in Germany, between 2013 and 2015. For this study, we extracted a subset of participants with no substance use or abuse, or familiarity with alcohol dependence, and past or present psychopathologies diagnosis at screening. The subset was therefore composed of 79 subjects ( 23 females; age range: 20-35 years; mean education: 12.39 years). We extracted raw data from structural MRI scans (T1 Weighted MP2RAGE) and functional MRI scans (rs-fMRI). With regards to behavioural data, the scores of the following questionnaires were selected: teiQUE-SF (Trait El Questionnaire - Short Form), ERQ (ER Questionnaire) and CERQ (Cognitive ER Questionnaire).

\section{MRI Data Acquisition}

Structural and functional MRI data in the LEMON dataset were acquired with a 3 Tesla MRI scanner (Verio, Siemens Healthcare $\mathrm{GmbH}$ ). During the acquisition, subjects were asked to remain awake with open eyes while looking at a low-contrast fixation cross. For our analyses we considered a BOLD rs-fMRI scan, using T2-weighted multiband $E P{ }^{*}$ sequence $\left(T R=1400 \mathrm{~ms}, T E=30 \mathrm{~ms}\right.$, flip angle $=69^{\circ}$, echo spacing $=0.67$ ms number of volumes $=657$, voxel size $=2.3 \mathrm{~mm}$, total acquisition time was $15 \min 30 \mathrm{~s}$ ) and T1-weighted structural volumes acquired using MP2RAGE sequence (TR=5000 ms, TE=2.92 ms, $\mathrm{TI} 1=700 \mathrm{~ms}, \mathrm{TI} 2=2500 \mathrm{~ms}$, FOV=256 mm, voxel size $=1 \mathrm{~mm}$ isotropic) The structural volumes were acquired with 176 slices interspersed during $8 \mathrm{~min} 22$ s of scanning ${ }^{92}$.

\section{Questionnaires}

Behavioural data used in the present study consist of 3 self-administered questionnaires. The Trait EI Questionnaire Short-Form (TEIQue - SF) was used to measure El as a personality trait ${ }^{61}$. The questionnaire, administered in the German version ${ }^{93}$ and measures four factors: well-being, self-control, emotionality, sociability, and a total index of trait El that consists of the average of the above factors. The Emotion Regulation Questionnaire (ERQ), adopted to measure the interindividual differences in the 
frequency of use of ER strategies ${ }^{12}$. The ERQ questionnaire was administered in the German version ${ }^{94}$, and consists of 10 items that allow a measure of the tendency to use two strategies of ER: cognitive reappraisal (6 items) and suppression of emotions (4 items). The Cognitive Emotion Regulation Questionnaire (CERQ), administered in the German version ${ }^{62}$, was used to measure the cognitive strategies that characterize the individual's style of ER. The questionnaire consists of 36 items divided into 9 scales that measure five strategies defined as adaptive: acceptance, positive refocusing, refocus on planning, focus on positivity, putting into perspective, and four non-adaptive strategies: self-blame, blaming others, rumination, catastrophizing ${ }^{95}$.

\section{Behavioural Analyses}

To test the first hypothesis of the present study, namely that trait El predicts ER style, we implemented two different analyses using SPSS Statistics for Windows, version 25.0 (SPSS Inc., Chicago, III., USA). In the first we implemented a Multivariate Linear Regression (MLR) with ERQ and CERQ questionnaires as dependent variables, while the total trait El was included as a predictor. Moreover, to assess the effect of every subscale of trait El, we next implemented a Multivariate Multiple Linear Regression (MMLR) with each subscale of the ERQ and CERQ questionnaires as dependent variables, and the four factors of the TeiQue-SF as predictors. Type I error was controlled by applying false discovery rate (FDR) correction to $\mathrm{p}$ values.

\section{Neuroimaging Analyses}

Pre-processing and functional connectivity analysis were conducted using CONN MATLAB Toolbox (version 18b) ${ }^{96}$. Firstly, we implemented CONN's default pre-processing pipeline using SMP12 default parameters which includes the following steps: functional realignment and unwarping, translation and centering, functional outlier detection (conservative settings), functional direct segmentation and normalization ( $1 \mathrm{~mm}$ resolution), structural translation, and centering, structural segmentation and normalization (2,4 $\mathrm{mm}$ resolution), functional and structural smoothing (spatial convolution with Gaussian kernel $8 \mathrm{~mm}$ ). Next, the denoising phase was implemented. The objective of this phase is the identification and elimination of confounding variables and artefacts from the estimated BOLD signal. Briefly, these factors are derived from three different sources (BOLD signal coming from white matter or cerebrospinal fluid masks, parameters and outliers defined in the pre-processing step, and an estimate of the pre-processing the subjects' motion parameters) ${ }^{97}$. Once identified, the factors are entered into a regression model (Ordinary Least Squares) as covariates. Finally, a 0.0008-0.09 Hz temporal band-pass filter standard for resting-state connectivity analyses was applied to the time series. Next, the functional connectivity analysis has been implemented. For this study, we chose to use a data-driven approach by implementing a group-Independent Component Analysis (group-ICA). The group-ICA implemented by CONN includes the following steps: pre-conditioning variance normalization, concatenation of the BOLD signal along the temporal dimension, dimensionality reduction at the group level, fast-ICA for spatial component estimation, and the back-projection for spatial estimation on the individual subject ${ }^{97}$. The number of independent components to be identified was set to $20^{98}$. In order to separate noise 
components from the underlying resting-state networks, every identified IC were visually inspected. Subsequently, one out of 20 ICs (IC17), due to its extent, did not allow for the delineation of specific areas and was discarded from the following analyses. Finally, we extracted the temporal variability of each IC's, calculated in CONN as SD of each BOLD time-series ${ }^{97}$. Type I error was controlled using cluster-sizebased false discovery rate (FDR) correction $(p<0.05$, voxel thresholded at $p<0.001$ (Worsley et al., 1996), within each analysis). Next, to assess the relationship between IC's temporal variability and both trait EI and ER, we implemented 2 different analysis by using SPSS Statistics for Windows, version 25.0 (SPSS Inc., Chicago, III., USA). Firstly, to address which of the 20 identified IC's predicted the trait El, we tested the individual explanatory variables effect (IC's BOLD variability values) on the TEIQue-SF factors and total index by using a Multiple Linear Regression model (Ordinary Least Squares) with a stepwise method (forward) for each dependent variable. Because we do not expect that all the identified components to be related to the investigated construct, we chose a method of fitting regression models in which the choice of predictor variables is made by an automatic procedure. This methodology consists of testing the incremental predictivity of the model: starting from a model with no predictor, each explanatory variable is added to the model and compared to the inclusion or exclusion threshold criterion (in our case predictor's p-value $<=0.05$ for inclusion) until the model reaches its maximum predictivity. Finally, the BOLD temporal variability of IC's that resulted to be significant predictors of trait El in the previous analysis were entered a Multivariate Multiple Regression (MMR) as independent variables to predict ER scores (ERQ and CERQ subscales). To avoid multiple comparisons issues, type I error was controlled applying false discovery rate correction (FDR) within each analysis.

\section{Declarations}

\section{Data Availability}

The complete LEMON Data can be accessed via Gesellschaft für wissenschaftliche Datenverarbeitung mbH Göttingen (GWDG) https://www.gwdg.de/. Raw and preprocessed data at this location is accessible through web browser https://ftp.gwdg.de/pub/misc/MPI-Leipzig_Mind-Brain-Body-LEMON/ and a fast FTP connection (ftp://ftp.gwdg.de/pub/misc/MPI-Leipzig_Mind-Brain-Body-LEMON/). In the case the location of the data changes in the future, the location of the dataset can be resolved with PID 21.11101/0000-0007-C379-5 (e.g. http://hdl.handle.net/21.11101/0000-0007-C379-5).

\section{Author contributions}

F.Z. conceived the work, analysed data, wrote the manuscript. B.M. wrote the manuscript. A.G. prepared figure and reviewed the manuscript.

\section{Competing Interests}

The authors declare no competing interests.

\section{References}


1. Campbell-Sills, L. \& Barlow, D. H. Incorporating Emotion Regulation into Conceptualizations and Treatments of Anxiety and Mood Disorders. in Handbook of emotion regulation. 542-559 (The Guilford Press, 2007).

2. Cole, P. M., Martin, S. E. \& Dennis, T. A. Emotion Regulation as a Scientific Construct: Methodological Challenges and Directions for Child Development Research. Child Dev. 75, 317-333 (2004).

3. Grecucci, A., Sulpizio, S., Tommasello, E., Vespignani, F. \& Job, R. Seeing emotions, reading emotions: Behavioral and ERPs evidence of the regulation of pictures and words. PLOS ONE 14, e0209461 (2019).

4. Gross, J. J. \& Thompson, R. A. Emotion Regulation: Conceptual Foundations. in Handbook of emotion regulation. 3-24 (The Guilford Press, 2007).

5. Aldao, A., Nolen-Hoeksema, S. \& Schweizer, S. Emotion-regulation strategies across psychopathology: A meta-analytic review. Clin. Psychol. Rev. 30, 217-237 (2010).

6. Nolen-Hoeksema, S., Wisco, B. E. \& Lyubomirsky, S. Rethinking Rumination. Perspect. Psychol. Sci. 3, 400-424 (2008).

7. Bonanno, G. A. \& Burton, C. L. Regulatory Flexibility: An Individual Differences Perspective on Coping and Emotion Regulation. Perspect. Psychol. Sci. 8, 591-612 (2013).

8. Eftekhari, A., Zoellner, L. A. \& Vigil, S. A. Patterns of emotion regulation and psychopathology. Anxiety Stress Coping 22, 571-586 (2009).

9. Sheppes, G., Suri, G. \& Gross, J. J. Emotion Regulation and Psychopathology. Annu. Rev. Clin. Psychol. 11, 379-405 (2015).

10. Brans, K., Koval, P., Verduyn, P., Lim, Y. L. \& Kuppens, P. The regulation of negative and positive affect in daily life. Emotion 13, 926-939 (2013).

11. Nezlek, J. B. \& Kuppens, P. Regulating Positive and Negative Emotions in Daily Life. J. Pers. 76, 561580 (2008).

12. Gross, J. J. \& John, O. P. Individual differences in two emotion regulation processes: Implications for affect, relationships, and well-being. J. Pers. Soc. Psychol. 85, 348-362 (2003).

13. Dryman, M. T. \& Heimberg, R. G. Emotion regulation in social anxiety and depression: a systematic review of expressive suppression and cognitive reappraisal. Clin. Psychol. Rev. 65, 17-42 (2018).

14. Ehring, T., Tuschen-Caffier, B., Schnülle, J., Fischer, S. \& Gross, J. J. Emotion regulation and vulnerability to depression: Spontaneous versus instructed use of emotion suppression and reappraisal. Emotion 10, 563-572 (2010).

15. Webb, T. L., Miles, E. \& Sheeran, P. Dealing with feeling: A meta-analysis of the effectiveness of strategies derived from the process model of emotion regulation. Psychol. Bull. 138, 775-808 (2012).

16. Schönfelder, S., Kanske, P., Heissler, J. \& Wessa, M. Time course of emotion-related responding during distraction and reappraisal. Soc. Cogn. Affect. Neurosci. 9, 1310-1319 (2014). 
17. Morawetz, C., Bode, S., Baudewig, J. \& Heekeren, H. R. Effective amygdala-prefrontal connectivity predicts individual differences in successful emotion regulation. Soc. Cogn. Affect. Neurosci. 12, 569-585 (2017).

18. Peña-Sarrionandia, A., Mikolajczak, M. \& Gross, J. J. Integrating emotion regulation and emotional intelligence traditions: a meta-analysis. Front. Psychol. 6, 160 (2015).

19. Hughes, D. J. \& Evans, T. R. Comment: Trait El Moderates the Relationship Between Ability El and Emotion Regulation. Emot. Rev. 8, 331-332 (2016).

20. Smith, R., Killgore, W. D. S. \& Lane, R. D. The structure of emotional experience and its relation to trait emotional awareness: A theoretical review. Emotion 18, 670-692 (2018).

21. Hughes, D. J. \& Evans, T. R. Putting 'Emotional Intelligences' in Their Place: Introducing the Integrated Model of Affect-Related Individual Differences. Front. Psychol. 9, 2155 (2018).

22. Petrides, K. V. et al. Developments in Trait Emotional Intelligence Research. Emot. Rev. 8, 335-341 (2016).

23. Bucich, M. \& MacCann, C. Emotional Intelligence and Day-To-Day Emotion Regulation Processes: Examining Motives for Social Sharing. Personal. Individ. Differ. 137, 22-26 (2019).

24. Davis, S. K. \& Humphrey, N. Ability Versus Trait Emotional Intelligence: Dual Influences on Adolescent Psychological Adaptation. J. Individ. Differ. 35, 54-62 (2014).

25. Côté, S. \& Hideg, I. The ability to influence others via emotion displays: A new dimension of emotional intelligence. Organ. Psychol. Rev. 1, 53-71 (2011).

26. Mayer, J. D., Caruso, D. R. \& Salovey, P. The Ability Model of Emotional Intelligence: Principles and Updates. Emot. Rev. 8, 290-300 (2016).

27. Mikolajczak, M., Nelis, D., Hansenne, M. \& Quoidbach, J. If you can regulate sadness, you can probably regulate shame: Associations between trait emotional intelligence, emotion regulation and coping efficiency across discrete emotions. Personal. Individ. Differ. 44, 1356-1368 (2008).

28. Killgore, W. D. S. \& Yurgelun-Todd, D. A. Neural correlates of emotional intelligence in adolescent children. Cogn. Affect. Behav. Neurosci. 7, 140-151 (2007).

29. Kreifelts, B., Ethofer, T., Huberle, E., Grodd, W. \& Wildgruber, D. Association of trait emotional intelligence and individual $\mathrm{fMRl}$-activation patterns during the perception of social signals from voice and face. Hum. Brain Mapp. 31, 979-991 (2009).

30. Mestre, J. M., MacCann, C., Guil, R. \& Roberts, R. D. Models of Cognitive Ability and Emotion Can Better Inform Contemporary Emotional Intelligence Frameworks. Emot. Rev. 8, 322-330 (2016).

31. Banks, S. J., Eddy, K. T., Angstadt, M., Nathan, P. J. \& Phan, K. L. Amygdala-frontal connectivity during emotion regulation. Soc. Cogn. Affect. Neurosci. 2, 303-312 (2007).

32. Buhle, J. T. et al. Cognitive Reappraisal of Emotion: A Meta-Analysis of Human Neuroimaging Studies. Cereb. Cortex 24, 2981-2990 (2014).

33. Carlson, J. M. \& Mujica-Parodi, L. R. A disposition to reappraise decreases anterior insula reactivity during anxious anticipation. Biol. Psychol. 85, 383-385 (2010). 
34. Drabant, E. M., McRae, K., Manuck, S. B., Hariri, A. R. \& Gross, J. J. Individual Differences in Typical Reappraisal Use Predict Amygdala and Prefrontal Responses. Biol. Psychiatry 65, 367-373 (2009).

35. Grecucci, A., Giorgetta, C., Bonini, N. \& Sanfey, A. G. Reappraising social emotions: the role of inferior frontal gyrus, temporo-parietal junction and insula in interpersonal emotion regulation. Front. Hum. Neurosci. 7, (2013).

36. Ochsner, K. N., Silvers, J. A. \& Buhle, J. T. Functional imaging studies of emotion regulation: a synthetic review and evolving model of the cognitive control of emotion: Functional imaging studies of emotion regulation. Ann. N. Y. Acad. Sci. 1251, E1-E24 (2012).

37. Giuliani, N. R., Drabant, E. M. \& Gross, J. J. Anterior cingulate cortex volume and emotion regulation: Is bigger better? Biol. Psychol. 86, 379-382 (2011).

38. Hermann, A., Bieber, A., Keck, T., Vaitl, D. \& Stark, R. Brain structural basis of cognitive reappraisal and expressive suppression. Soc. Cogn. Affect. Neurosci. 9, 1435-1442 (2014).

39. Killgore, W. D. S. et al. Gray matter correlates of Trait and Ability models of emotional intelligence. NeuroReport 23, 551-555 (2012).

40. Sala-Llonch, R. et al. Brain connectivity during resting state and subsequent working memory task predicts behavioural performance. Cortex 48, 1187-1196 (2012).

41. Smith, S. M. et al. Correspondence of the brain's functional architecture during activation and rest. Proc. Natl. Acad. Sci.<bvertical-align:super;>106</bvertical-align:super;>, 13040-13045 (2009).

42. Tavor, I. et al. Task-free MRI predicts individual differences in brain activity during task performance. Science 352, 216-220 (2016).

43. Kaiser, R. H., Andrews-Hanna, J. R., Wager, T. D. \& Pizzagalli, D. A. Large-Scale Network Dysfunction in Major Depressive Disorder: A Meta-analysis of Resting-State Functional Connectivity. JAMA Psychiatry 72, 603 (2015).

44. Veer, I. M. Whole brain resting-state analysis reveals decreased functional connectivity in major depression. Front. Syst. Neurosci. 4, (2010).

45. Pan, W. et al. Identifying the Core Components of Emotional Intelligence: Evidence from Amplitude of Low-Frequency Fluctuations during Resting State. PLOS ONE 9, e111435 (2014).

46. Killgore, W. D. S. et al. Emotional intelligence is associated with connectivity within and between resting state networks. Soc. Cogn. Affect. Neurosci. 12, 1624-1636 (2017).

47. Takeuchi, H. et al. Resting state functional connectivity associated with trait emotional intelligence. Neurolmage 83, 318-328 (2013).

48. Picó-Pérez, M. et al. Dispositional use of emotion regulation strategies and resting-state corticolimbic functional connectivity. Brain Imaging Behav. 12, 1022-1031 (2018).

49. Uchida, M. et al. Emotion regulation ability varies in relation to intrinsic functional brain architecture. Soc. Cogn. Affect. Neurosci. 10, 1738-1748 (2015).

50. Dörfel, D., Gärtner, A. \& Scheffel, C. Resting State Cortico-Limbic Functional Connectivity and Dispositional Use of Emotion Regulation Strategies: A Replication and Extension Study. Front. Behav. 
Neurosci. 14, 128 (2020).

51. Grecucci, A., Sorella, S., Consolini, J. Decoding Individual Differences in Controlling, Expressing and Inhibiting Anger from Structural Brain Networks: a Supervised Machine Learning Approach. Brain Sciences (In press).

52. Bijsterbosch, J. Introduction to resting state fMRI functional connectivity. (Oxford University Press, 2017).

53. Garrett, D. D., Kovacevic, N., McIntosh, A. R. \& Grady, C. L. Blood Oxygen Level-Dependent Signal Variability Is More than Just Noise. J. Neurosci. 30, 4914-4921 (2010).

54. Garrett, D. D. et al. Moment-to-moment brain signal variability: A next frontier in human brain mapping? Neurosci. Biobehav. Rev. 37, 610-624 (2013).

55. Vakorin, V. A., Lippe, S. \& McIntosh, A. R. Variability of Brain Signals Processed Locally Transforms into Higher Connectivity with Brain Development. J. Neurosci. 31, 6405-6413 (2011).

56. Guassi Moreira, J. F., McLaughlin, K. A. \& Silvers, J. A. Spatial and temporal cortical variability track with age and affective experience during emotion regulation in youth. Dev. Psychol. 55, 1921-1937 (2019).

57. Aldao, A., Sheppes, G. \& Gross, J. J. Emotion Regulation Flexibility. Cogn. Ther. Res. 39, 263-278 (2015).

58. Heller, A. S. \& Casey, B. J. The neurodynamics of emotion: delineating typical and atypical emotional processes during adolescence. Dev. Sci. 19, 3-18 (2016).

59. Sorella, S., Vellani, V., Siugzdaite, R., Feraco, P. \& Grecucci, A. Structural and functional brain networks of individual differences in trait anger and anger control: An unsupervised machine learning study. Eur. J. Neurosci. ejn.15537 (2021) doi:10.1111/ejn.15537.

60. Armbruster-Genç, D. J. N., Ueltzhöffer, K. \& Fiebach, C. J. Brain Signal Variability Differentially Affects Cognitive Flexibility and Cognitive Stability. J. Neurosci. 36, 3978-3987 (2016).

61. Petrides, K. V. Psychometric Properties of the Trait Emotional Intelligence Questionnaire (TEIQue). in Assessing Emotional Intelligence (eds. Parker, J. D. A., Saklofske, D. H. \& Stough, C.) 85-101 (Springer US, 2009). doi:10.1007/978-0-387-88370-0_5.

62. Loch, N., Hiller, W. \& Witthöft, M. Der Cognitive Emotion Regulation Questionnaire (CERQ): Erste teststatistische Überprüfung einer deutschen Adaption. Z. Für Klin. Psychol. Psychother. 40, 94-106 (2011).

63. Fernández-Berrocal, P. \& Extremera, N. Ability Emotional Intelligence, Depression, and Well-Being. Emot. Rev. 8, 311-315 (2016).

64. Hertel, J., Schütz, A. \& Lammers, C.-H. Emotional intelligence and mental disorder. J. Clin. Psychol. 65, 942-954 (2009).

65. Hasegawa, A., Somatori, K., Nishimura, H., Hattori, Y. \& Kunisato, Y. Depression, Rumination, and Impulsive Action: A Latent Variable Approach to Behavioral Impulsivity. J. Psychol. 155, 717-737 (2021). 
66. Bugay-Sökmez, A., Manuoğlu, E., Coşkun, M. \& Sümer, N. Predictors of rumination and co-rumination: the role of attachment dimensions, self-compassion and self-esteem. Curr. Psychol. (2021) doi:10.1007/s12144-021-01799-0.

67. Lanteigne, D. M., Flynn, J. J., Eastabrook, J. M. \& Hollenstein, T. Discordant patterns among emotional experience, arousal, and expression in adolescence: Relations with emotion regulation and internalizing problems. Can. J. Behav. Sci. Rev. Can. Sci. Comport. 46, 29-39 (2014).

68. Velotti, P., Garofalo, C., Bottazzi, F. \& Caretti, V. Faces of Shame: Implications for Self-Esteem, Emotion Regulation, Aggression, and Well-Being. J. Psychol. 151, 171-184 (2017).

69. Gross, J. J. The Emerging Field of Emotion Regulation: An Integrative Review. Rev. Gen. Psychol. 2 , 271-299 (1998).

70. Eisenberg, N. \& Fabes, R. A. The relation of young children's vicarious emotional responding to social competence, regulation, and emotionality. Cogn. Emot. 9, 203-228 (1995).

71. Takeuchi, H. et al. Regional gray matter density associated with emotional intelligence: Evidence from voxel-based morphometry. Hum. Brain Mapp. 32, 1497-1510 (2011).

72. Grecucci, A., Rubicondo, D., Siugzdaite, R., Surian, L. \& Job, R. Uncovering the Social Deficits in the Autistic Brain. A Source-Based Morphometric Study. Front. Neurosci. 10, (2016).

73. Gallese, V. \& Keysers, C. Mirror neurons: A sensorimotor representation system. Behav. Brain Sci. 24, 983-984 (2001).

74. Pineda, J. A. Sensorimotor cortex as a critical component of an 'extended' mirror neuron system: Does it solve the development, correspondence, and control problems in mirroring? Behav. Brain Funct. 4, 47 (2008).

75. Damasio, A. R. et al. Subcortical and cortical brain activity during the feeling of self-generated emotions. Nat. Neurosci. 3, 1049-1056 (2000).

76. Critchley, H. D., Wiens, S., Rotshtein, P., Öhman, A. \& Dolan, R. J. Neural systems supporting interoceptive awareness. Nat. Neurosci. 7, 189-195 (2004).

77. Bar-On, R. Exploring the neurological substrate of emotional and social intelligence. Brain 126, 1790-1800 (2003).

78. Grecucci, A., Giorgetta, C., van't Wout, M., Bonini, N. \& Sanfey, A. G. Reappraising the Ultimatum: an fMRI Study of Emotion Regulation and Decision Making. Cereb. Cortex 23, 399-410 (2013).

79. Lisowska, A. et al. Joint Pairing and Structured Mapping of Convolutional Brain Morphological Multiplexes for Early Dementia Diagnosis. Brain Connect. 9, 22-36 (2019).

80. Papousek, l. et al. The capacity for generating cognitive reappraisals is reflected in asymmetric activation of frontal brain regions. Brain Imaging Behav. 11, 577-590 (2017).

81. Morawetz, C. et al. Multiple large-scale neural networks underlying emotion regulation. Neurosci. Biobehav. Rev. 116, 382-395 (2020).

82. Burklund, L. J., Creswell, J. D., Irwin, M. R. \& Lieberman, M. D. The common and distinct neural bases of affect labeling and reappraisal in healthy adults. Front. Psychol. 5, (2014). 
83. Kassam, K. S., Markey, A. R., Cherkassky, V. L., Loewenstein, G. \& Just, M. A. Identifying Emotions on the Basis of Neural Activation. PLoS ONE 8, e66032 (2013).

84. Satpute, A. B. \& Lindquist, K. A. At the Neural Intersection Between Language and Emotion. Affect. Sci. 2, 207-220 (2021).

85. Murakami, H. et al. Neural Networks for Mindfulness and Emotion Suppression. PLOS ONE 10, e0128005 (2015).

86. Hudetz, A. G., Liu, X. \& Pillay, S. Dynamic Repertoire of Intrinsic Brain States Is Reduced in PropofolInduced Unconsciousness. Brain Connect. 5, 10-22 (2015).

87. Stoica, T. \& Depue, B. Shared Characteristics of Intrinsic Connectivity Networks Underlying Interoceptive Awareness and Empathy. Front. Hum. Neurosci. 14, 571070 (2020).

88. Garrett, D. D., Kovacevic, N., McIntosh, A. R. \& Grady, C. L. The Importance of Being Variable. J. Neurosci. 31, 4496-4503 (2011).

89. Petroni, A. et al. The Variability of Neural Responses to Naturalistic Videos Change with Age and Sex. eneuro <bvertical-align:super;>5</bvertical-align:super;>, ENEURO.0244-17.2017 (2018).

90. Gross, J. J. The Extended Process Model of Emotion Regulation: Elaborations, Applications, and Future Directions. Psychol. Inq. 26, 130-137 (2015).

91. Elliott, M. L. et al. What Is the Test-Retest Reliability of Common Task-Functional MRI Measures? New Empirical Evidence and a Meta-Analysis. Psychol. Sci. 31, 792-806 (2020).

92. Babayan, A. et al. A mind-brain-body dataset of MRI, EEG, cognition, emotion, and peripheral physiology in young and old adults. Sci. Data 6, 180308 (2019).

93. Freudenthaler, H. H., Neubauer, A. C., Gabler, P., Scherl, W. G. \& Rindermann, H. Testing and validating the trait emotional intelligence questionnaire (TEIQue) in a German-speaking sample. Personal. Individ. Differ. 45, 673-678 (2008).

94. Abler, B. \& Kessler, H. Emotion Regulation Questionnaire - Eine deutschsprachige Fassung des ERQ von Gross und John. Diagnostica 55, 144-152 (2009).

95. Garnefski, N., Kraaij, V. \& Spinhoven, P. Negative life events, cognitive emotion regulation and emotional problems. Personal. Individ. Differ. 30, 1311-1327 (2001).

96. Whitfield-Gabrieli, S. \& Nieto-Castanon, A. Conn: A Functional Connectivity Toolbox for Correlated and Anticorrelated Brain Networks. Brain Connect. 2, 125-141 (2012).

97. Nieto-Castanon, A. Handbook of functional connectivity magnetic resonance imaging methods in CONN. (Hilbert Press, 2020).

98. Abou Elseoud, A. et al. Group-ICA Model Order Highlights Patterns of Functional Brain Connectivity. Front. Syst. Neurosci. 5, (2011).

\section{Figures}




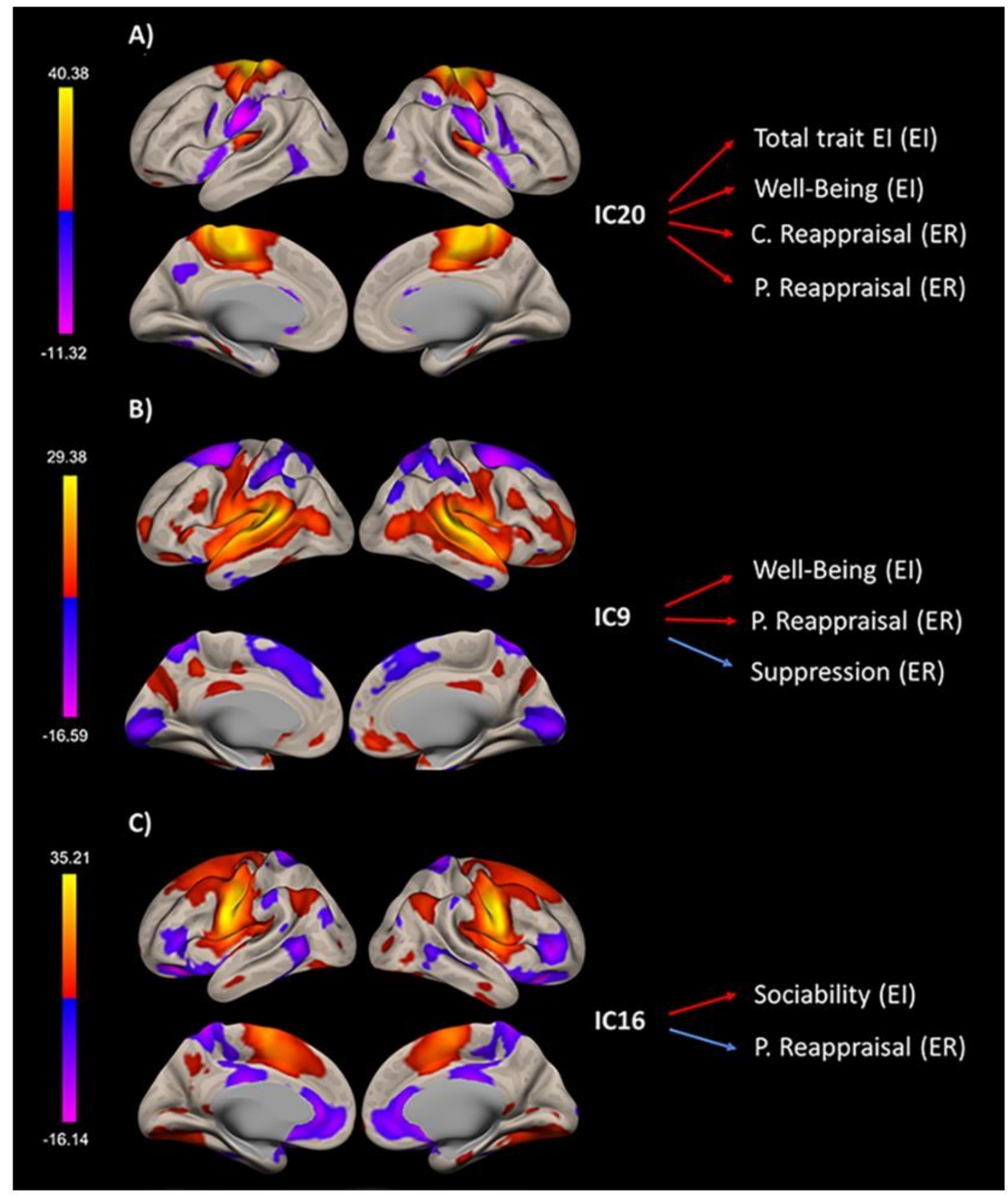

\section{Figure 1}

Representation of the ICs shared by trait El and ER. A) IC20; B) IC9; C) IC16. Left of the panel: 3D inflated brain model for the three ICs (cluster significance $p F D R<0.05$ and voxel significance $p F D R<0.001$ ). Color bar represents positive $t$-values in orange and negative $t$-values in blue. Right of the panel: Relation between the ICs and the subscales of trait El and ER questionnaires. In red, the positive relation; In blue, the negative relation. C. Reappraisal $=$ Cognitive Reappraisal; P. Reappraisal = Positive Reappraisal 